\title{
Enantioselective synthesis
} and selective monofunctionalization of (4R,6R)-4,6-dihydroxy-2,8dioxabicyclo[3.3.0]octane

Bruno Linclau, ${ }^{*}$ Martin J. Jeffery, ${ }^{\ddagger}$ Solen Josse, ${ }^{\perp}$ and Cyrille Tomassi

University of Southampton, School of Chemistry, Highfield, Southampton SO17 1BJ

Bruno.linclau@soton.ac.uk

\section{Supporting Information}

I. Reaction procedures and characterisation data for 5, 16, 17, 25a, 26b: p S2-S5.

II. X-ray structure of 5: $\mathrm{p} \mathrm{S6}$.

III. Stereochemical confirmation of 17: p S7.

IV. X-ray structures of the paranitrophenolates of $\mathbf{2 5 b}, \mathbf{2 6 \mathbf { b }}$ : p S8.

V. Copies of ${ }^{1} \mathrm{H}$ and ${ }^{13} \mathrm{C}$ NMR spectra of 5, 16, 17, 25a, 26b: p S9-S13.

\section{General Methods}

Arabitol was obtained from CMS Chemicals, and used without further purification. THF was distilled from $\mathrm{Na}$ /benzophenone immediately prior to use. Extra dry DMF (water $<50 \mathrm{ppm}$ ) was purchased from commercial sources. Glassware was flame-dried immediately before use. All reactions were performed under inert atmosphere. Column chromatography was performed on 230-400 mesh silica gel. Reactions were monitored by TLC (Merck) with alkaline $\mathrm{KMnO}_{4}$ and anisaldehyde dyes.

Data for all X-ray structures were collected on a Bruker Nonius KappaCCD mounted at the window of a Mo FR591 rotating anode. Standard data reduction, solution and refinement procedures were followed. 


\section{$\underline{\text { I. Reaction procedures and characterisation data }}$}

(4R,6R)-4,6-dihydroxy-2,8-dioxa-bicyclo[3.3.0]octane (5)
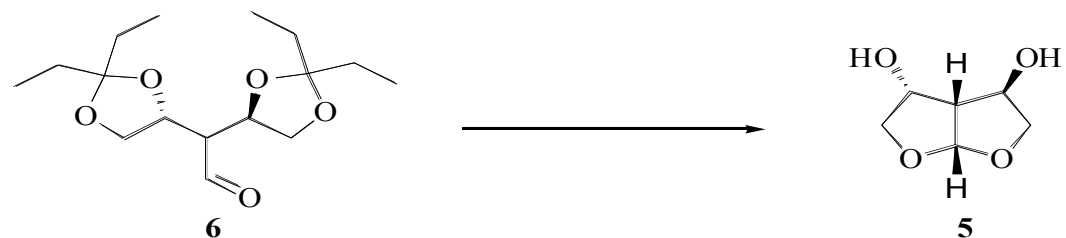

To a stirred solution of aldehyde $6(13.29 \mathrm{~g}, 44.2 \mathrm{mmol})$ in $130 \mathrm{~mL}$ of dichloromethane at room temperature was added $14.3 \mathrm{~mL}$ of a mixture of trifluoroacetic acid and water (9:1 ; $\mathrm{v} / \mathrm{v})$. After $30 \mathrm{~min}$ the solvent was removed in vacuo and the crude was co-evaporated with toluene. The purification by column chromatograpy (dichloromethane/methanol 9:1) gave the title compound $\mathbf{5}$ as a white solid (5.79 g, 90\%).

Formula $\mathrm{C}_{6} \mathrm{H}_{10} \mathrm{O}_{4}$; Mw 146.14; Mp 116- $117{ }^{\circ} \mathrm{C}$ (hexane/acetone); $\mathbf{R}_{\mathbf{f}} 0.24\left(\mathrm{CH}_{2} \mathrm{Cl}_{2} / \mathrm{MeOH}\right.$ 90:10); $[\alpha]_{\mathbf{D}}+45.8\left(c 0.61, \mathrm{MeOH}, 24{ }^{\circ} \mathrm{C}\right.$ ); IR (solid) 3381 (br s), 3323 (br s), 2988 (w), 2962 (w), $2876(\mathrm{w}), 1489$ (v), 1428 (v), 1339 (w), 1304 (w), 1272 (w), 1121 (s), 1089 (w), 999 (s), and $968(\mathrm{~s}) \mathrm{cm}^{-1}{ }^{1} \mathbf{H}$ NMR (400 MHz, DMSO-d $\left.{ }^{6}\right) \delta 5.62(1 \mathrm{H}, \mathrm{d}, J=5.5 \mathrm{~Hz}), 5.22(1 \mathrm{H}, \mathrm{d}, J$ $=4.5 \mathrm{~Hz}), 4.85(1 \mathrm{H}, \mathrm{d}, J=4.5 \mathrm{~Hz}), 4.43(1 \mathrm{H}, \mathrm{t}, J=4.0 \mathrm{~Hz}), 4.29(1 \mathrm{H}, \mathrm{m}), 3.79(1 \mathrm{H}, \mathrm{d}, J=$ $9.5 \mathrm{~Hz}), 3.78(1 \mathrm{H}, \mathrm{dd}, J=9.0,2.5 \mathrm{~Hz}), 3.68(1 \mathrm{H}, \mathrm{d}, J=9.5 \mathrm{~Hz}), 3.28(1 \mathrm{H}, \mathrm{m})$, and 2.57 (1 $\mathrm{H}, \mathrm{dd}, J=9.0,5.0 \mathrm{~Hz}) ;{ }^{13} \mathbf{C}$ NMR + DEPT $\left(100 \mathrm{MHz}, \mathrm{DMSO}-\mathrm{d}^{6}\right) \delta 108.7(\mathrm{CH}), 77.1\left(\mathrm{CH}_{2}\right)$, $72.3\left(\mathrm{CH}_{2}\right), 70.4(\mathrm{CH}), 68.5(\mathrm{CH})$, and $55.1(\mathrm{CH})$; EIMS $m / z(\%) 147\left((\mathrm{M}+\mathrm{H})^{+}, 8\right), 129((\mathrm{M}$ $\left.\left.-\mathrm{H}_{2} \mathrm{O}\right)^{+}, 8\right), 116(58), 87$ (67), and 69 (100); HRMS (EI) for $\mathrm{C}_{6} \mathrm{H}_{11} \mathrm{O}_{4}(\mathrm{M}+\mathrm{H})^{+}$calcd 147.06573, found 147.06544; Anal. Calcd for $\mathrm{C}_{6} \mathrm{H}_{10} \mathrm{O}_{4}$ : C, 49.41; H, 6.90. Found: C, 49.10; H, 7.01.

\section{2,2-bis((R)-2,2-diethyl-1,3-dioxolan-4-yl)ethanol (16)}

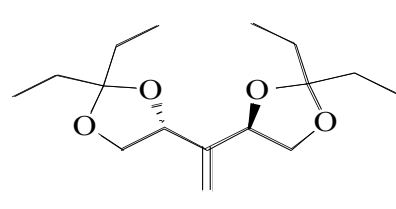

15

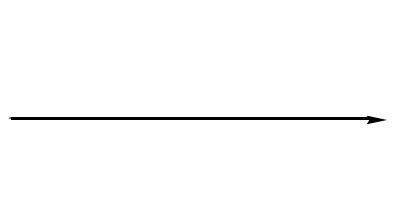

A solution of triethylborane $(10.2 \mathrm{~mL}, 1.0 \mathrm{M}$ in THF) and borane (1.7 $\mathrm{mL}, 1.0 \mathrm{M}$ in THF) was stirred at room temperature for $1 \mathrm{~h}$. A solution of the $\mathrm{C}_{2}$-symmetric alkene 15 (968 $\mathrm{mg}$, $3.40 \mathrm{mmol})$ in THF $(7 \mathrm{~mL})$ was added and the reaction mixture stirred for $2 \mathrm{~d}$. The reaction mixture was then carefully transferred via cannula into a stirred mixture of $\mathrm{NaOH}(\mathrm{aq}, 3$ M): $\mathrm{H}_{2} \mathrm{O}_{2}$ (aq, $27 \%$ wt.): $\mathrm{CH}_{2} \mathrm{Cl}_{2}\left(1: 1: 1,90 \mathrm{~mL}\right.$ ) at $0{ }^{\circ} \mathrm{C}$ and stirred for $2 \mathrm{~h}$. The layers were 
separated, and the aqueous layer extracted with $\mathrm{CH}_{2} \mathrm{Cl}_{2}(3 \times 30 \mathrm{~mL})$. The combined organic phases were dried over anhydrous $\mathrm{Na}_{2} \mathrm{SO}_{4}$, filtered and evaporated to give the crude product as a oil. Purification by column chromatography (hexane/acetone 85:15) gave the title compound 16 as a colourless oil (950 mg, $92 \%)$.

Formula $\mathrm{C}_{16} \mathrm{H}_{30} \mathrm{O}_{5} ; \mathbf{M w} 302.41 ; \mathbf{R}_{\mathbf{f}} 0.28$ (hexane/acetone 80:20); $[\boldsymbol{\alpha}]_{\mathbf{D}}-9.7$ (c 1.06, $\mathrm{CHCl}_{3}$, $23{ }^{\circ} \mathrm{C}$ ); IR (film) 3458 (br m), 2972 (s), 2936 (s), 2882 (s), 1464 (m), 1355 (m), 1173 (m), 1078 (s), and 1057 (s) $\mathrm{cm}^{-1}$; ${ }^{1} \mathbf{H}$ NMR $\left(300 \mathrm{MHz}, \mathrm{CDCl}_{3}\right) \delta$ 4.24-4.11 (2 H, m), 4.12 (1 H, dd, $J=8.1,5.9 \mathrm{~Hz}), 3.96(1 \mathrm{H}, \mathrm{td}, J=8.8,5.9 \mathrm{~Hz}), 3.74-3.66(3 \mathrm{H}, \mathrm{m}), 3.61(1 \mathrm{H}, \mathrm{dd}, J=8.8,8.1$ Hz), 2.63 (1 H, br s), 1.84 (1 H, m), 1.67-1.54 (8 H, m), 0.897 (3 H, t, J = 7.35 Hz), 0.890 (3 $\mathrm{H}, \mathrm{t}, J=7.35 \mathrm{~Hz}), 0.87(3 \mathrm{H}, \mathrm{t}, J=7.35 \mathrm{~Hz})$, and $0.86(3 \mathrm{H}, \mathrm{t}, J=7.35 \mathrm{~Hz}) ;{ }^{13} \mathbf{C ~ N M R ~ + ~}$ DEPT $\left(75 \mathrm{MHz}, \mathrm{CDCl}_{3}\right) \delta 112.7(\mathrm{C}), 112.2(\mathrm{C}), 78.2(\mathrm{CH}), 75.0(\mathrm{CH}), 70.3\left(\mathrm{CH}_{2}\right), 69.1$ $\left(\mathrm{CH}_{2}\right), 62.1\left(\mathrm{CH}_{2}\right), 48.0(\mathrm{CH}), 29.79\left(\mathrm{CH}_{2}\right), 29.67\left(\mathrm{CH}_{2}\right), 29.3\left(2 \times \mathrm{CH}_{2}\right), 8.18\left(\mathrm{CH}_{3}\right), 8.11$ $\left(\mathrm{CH}_{3}\right), 8.05\left(\mathrm{CH}_{3}\right)$, and $7.97\left(\mathrm{CH}_{3}\right)$.

CIMS $m / z(\%) 303\left((\mathrm{M}+\mathrm{H})^{+}, 4\right), 273\left(\left(\mathrm{M}-\mathrm{C}_{2} \mathrm{H}_{5}\right)^{+}, 7\right), 217$ (96), 187 (26) and 57 (100); HRMS (CI) for $\mathrm{C}_{16} \mathrm{H}_{31} \mathrm{O}_{5}(\mathrm{M}+\mathrm{H})^{+}$calcd 303.21715, found 303.21766.

(R)-2-[(R)-2,2-diethyl-1,3-dioxolan-4-yl)-4-(1-ethyl-propoxy)]butane-1,3-diol (17)

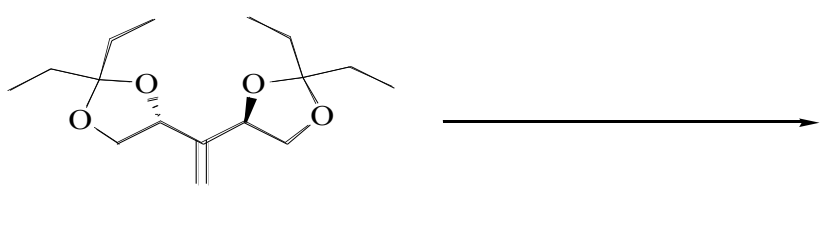

15

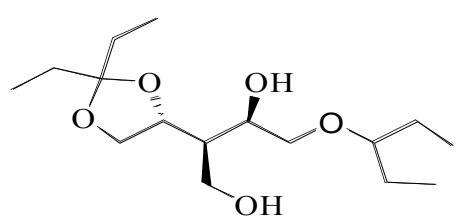

17

To a solution of the alkene $15(1.50 \mathrm{~g}, 5.27 \mathrm{mmol})$ in THF at $0^{\circ} \mathrm{C}$ was added borane $(52.8 \mathrm{~mL}$, $52.8 \mathrm{mmol}, 1 \mathrm{M}$ in THF). The solution was allowed to warm at room temperature and was stirred for two days. TLC analysis indicated that starting material still remained, so the temperature was raised to $40^{\circ} \mathrm{C}$ and the reaction mixture was stirred for one day. $\mathrm{CH}_{2} \mathrm{Cl}_{2}(100$ $\mathrm{mL}$ ) was added and a solution of $\mathrm{NaOH}$ (aq, 3M)/ $\mathrm{H}_{2} \mathrm{O}_{2}$ (aq, 27\% wt.) (1:1, $50 \mathrm{~mL}$ ) was added dropwise (CARE). This mixture was stirred for one hour. The layers were separated and the aqueous layer extracted with $\mathrm{CH}_{2} \mathrm{Cl}_{2}(2 \times 50 \mathrm{~mL})$. The organic phases were combined, dried over anhydrous $\mathrm{MgSO}_{4}$, filtered and the solvent removed in vacuo to give a colorless residue. Purification by column chromatography (hexane/ acetone 80:20) gave the title compound as a colourless oil as a single diastereoisomere (1.346 g, 84\%).

Formula $\mathrm{C}_{16} \mathrm{H}_{32} \mathrm{O}_{5} ; \mathbf{M w} 304.43 ; \mathbf{R}_{\mathbf{f}} 0.18$ (hexane/acetone 80:20); $[\boldsymbol{\alpha}]_{\mathbf{D}}-12.5$ (c 0.98, $\mathrm{CHCl}_{3}$, $24{ }^{\circ} \mathrm{C}$ ); IR (film) 3424 (br m), 2969 (s), 2937 (s), 2879 (s), 1463 (s), 1379 (m), 1355 (m), 1293 (w), 1273 (w), 1198 (m), 1173 (m), 1131 (s), 1079 (s), 1057 (s) cm ${ }^{-1}$; ${ }^{1} \mathbf{H}$ NMR (400 
$\left.\mathrm{MHz}, \mathrm{CDCl}_{3}\right) \delta 4.32(1 \mathrm{H}, \mathrm{td}, J=8.5,6.0 \mathrm{~Hz}), 4.15(1 \mathrm{H}, \mathrm{dd}, J=8.0,6.0 \mathrm{~Hz}), 4.08(1 \mathrm{H}, \mathrm{br}$ m), $3.77(1 \mathrm{H}, \mathrm{dd}, J=11.5,3.0 \mathrm{~Hz}), 3.64-3.59(3 \mathrm{H}, \mathrm{m}), 3.51(1 \mathrm{H}, \mathrm{dd}, J=9.5,6.5 \mathrm{~Hz}), 3.44$ (1 H, br s), 3.23-3.17 (2 H, m), 1.80 (1 H, ddt, $J=8.5,5.0,3.5 \mathrm{~Hz}), 1.66-1.56$ (4 H, m), 1.531.46 (4 H, m), 0.89-0.84 (12 H, m) ppm; $\left.{ }^{13} \mathbf{C ~ N M R ~ + ~ D E P T ~ ( 1 0 0 ~ M H z , ~ C D C l ~}{ }_{3}\right) \delta 112.5(\mathrm{C})$, $82.8(\mathrm{CH}), 75.3(\mathrm{CH}), 71.5(\mathrm{CH}), 71.0\left(\mathrm{CH}_{2}\right), 68.9\left(\mathrm{CH}_{2}\right), 60.7\left(\mathrm{CH}_{2}\right), 46.6(\mathrm{CH}), 29.8\left(\mathrm{CH}_{2}\right)$, $29.4\left(\mathrm{CH}_{2}\right), 25.64\left(\mathrm{CH}_{2}\right), 25.59\left(\mathrm{CH}_{2}\right), 9.4\left(\mathrm{CH}_{3}\right), 9.3\left(\mathrm{CH}_{3}\right), 8.1\left(\mathrm{CH}_{3}\right), 7.9\left(\mathrm{CH}_{3}\right)$ ppm; CIMS $m / z(\%) 305\left((\mathrm{M}+\mathrm{H})^{+}, 5\right), 275\left(\left(\mathrm{M}-\mathrm{C}_{2} \mathrm{H}_{5}\right)^{+}, 6\right), 219(82), 57$ (100); HRMS (CI) for $\mathrm{C}_{16} \mathrm{H}_{33} \mathrm{O}_{5}(\mathrm{M}+\mathrm{H})^{+}$calcd 305.2328, found 305.2334.

(4R, 6R)-6-dihydroxy-2,8-dioxa-4-triisopropylsilanyloxy-bicyclo[3.3.0]octane (5)
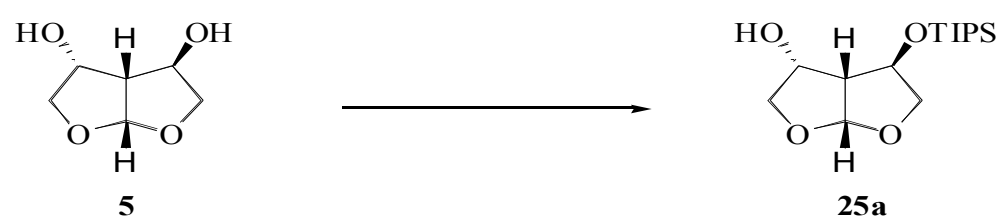

To a solution of the diol 5 (165 mg, $1.13 \mathrm{mmol})$, imidazole (230 mg, 3.39mmol) and DMAP (41 mg, $0.34 \mathrm{mmol})$ in DMF $(5 \mathrm{~mL})$ was added triisopropylsilyl chloride $(0.364 \mathrm{~mL}, 1.7$ $\mathrm{mmol}$ ) and was stirred at room temperature for $22 \mathrm{~h}$. The reaction was poured in brine (20 $\mathrm{mL})$ and the mixture was extracted with $\mathrm{Et}_{2} \mathrm{O}(3 \times 15 \mathrm{~mL})$. The organic layers were combined and dried over anhydrous $\mathrm{MgSO}_{4}$, filtered then evaporated. Purification of the resulting residue by column chromatography (hexane/acetone 75:25) gave the title compound as a colourless oil (273 mg, 80\%).

Formula $\mathrm{C}_{15} \mathrm{H}_{30} \mathrm{O}_{4} \mathrm{Si} ; \mathrm{Mw} 302.49 ; \mathbf{R}_{\mathbf{f}} 0.26$ (hexane/acetone 80:20); [ $\left.\boldsymbol{\alpha}\right]_{\mathbf{D}}+44.7$ (c 0.99, $\mathrm{CHCl}_{3}, 23{ }^{\circ} \mathrm{C}$ ); IR (film) 3444 (br m), 2943 (s), 2890 (s), 2866 (s), 1464 (s), 1383 (m), 1302 (w), 1244 (m), 1123 (s), 1044 (s), 997 (s) cm ${ }^{-1} ;{ }^{1} \mathbf{H}$ NMR (400 MHz, CDCl $\left.{ }_{3}\right) \delta 5.81$ (1 H, d, J $=5.0 \mathrm{~Hz}), 4.77(1 \mathrm{H}, \mathrm{d}, J=3.5 \mathrm{~Hz}), 4.48(1 \mathrm{H}, \mathrm{dt}, J=8.5,6.0 \mathrm{~Hz}), 4.03(1 \mathrm{H}, \mathrm{dd}, J=3.5,9.5$ $\mathrm{Hz}), 3.96(1 \mathrm{H}, \mathrm{dd}, J=9.5,6.0 \mathrm{~Hz}), 3.91(1 \mathrm{H}, \mathrm{d}, J=9.5 \mathrm{~Hz}), 3.60(1 \mathrm{H}, \mathrm{dd}, J=9.5,6.0 \mathrm{~Hz})$, $2.77(1 \mathrm{H}, \mathrm{dd}, J=5.0,8.5 \mathrm{~Hz}), 2.54(1 \mathrm{H}, \mathrm{br} \mathrm{s}), 1.08-1.04(21 \mathrm{H}, \mathrm{m}) \mathrm{ppm} ;{ }^{13} \mathbf{C}$ NMR + DEPT $\left(100 \mathrm{MHz}, \mathrm{CDCl}_{3}\right) \delta 109.3(\mathrm{CH}), 78.3\left(\mathrm{CH}_{2}\right), 73.7\left(\mathrm{CH}_{2}\right), 72.7(\mathrm{CH}), 69.7(\mathrm{CH}), 56.7(\mathrm{CH})$, $17.89\left(3 \mathrm{CH}_{3}\right), 17.87\left(3 \mathrm{CH}_{3}\right), 12.0(3 \mathrm{CH}) \mathrm{ppm}$; CIMS m/z (\%) $303\left((\mathrm{M}+\mathrm{H})^{+}, 10\right), 217(22)$, 199 (8), 173 (90), 148 (74) and 131 (100); HRMS (CI) for $\mathrm{C}_{15} \mathrm{H}_{31} \mathrm{O}_{4} \mathrm{Si}(\mathrm{M}+\mathrm{H})^{+}$calcd 303.1992, found 303.1999. 

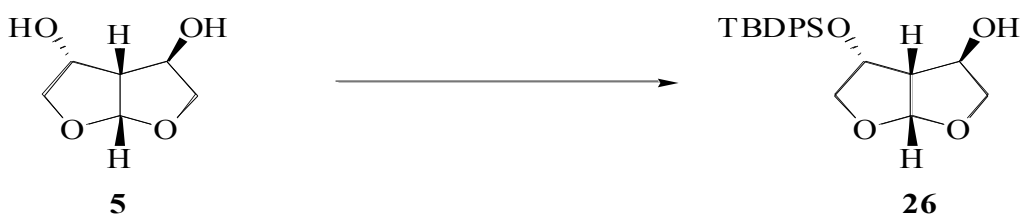

To a solution of the diol $5(1.996 \mathrm{~g}, 13.66 \mathrm{mmol})$, imidazole $(7.439 \mathrm{~g}, 109.26 \mathrm{mmol})$, DMAP $(1.225 \mathrm{~g}, 10.93 \mathrm{mmol})$ in DMF $(50 \mathrm{~mL})$ was added tert-butyldiphenylsilylchloride $(14.2 \mathrm{~mL}$, $54.64 \mathrm{mmol}$ ) and the reaction mixture was stirred at room temperature for one day. Once the reaction completed $200 \mathrm{~mL}$ of $\mathrm{Et}_{2} \mathrm{O}$ and $500 \mathrm{~mL}$ of water was added. The layers were separated and the organic layer washed with $300 \mathrm{~mL}$ of water and $300 \mathrm{~mL}$ of brine, then dried over anhydrous $\mathrm{Na}_{2} \mathrm{SO}_{4}$, filtered and the solvent evaporated to give the crude product as colorless oil. The crude product was dissolved in $100 \mathrm{~mL}$ of methanol and $\mathrm{NH}_{4} \mathrm{~F}(2.024 \mathrm{~g}$, $54.64 \mathrm{mmol}$ ) was added. The reaction was stirred at refluxing temperature $2.5 \mathrm{~h}$, and then the solvent was evaporated. Purification of the crude product by column chromatography (hexane/acetone 90:10, 85:15 then 100\% acetone) gave successively 28 (impure) and the title compound 26 as a colourless oil (2.651g, 50\%).

Formula $\mathrm{C}_{22} \mathrm{H}_{28} \mathrm{O}_{4} \mathrm{Si} ;$ Mw 384.54; $\mathbf{R}_{\mathbf{f}} 0.76$ (hexane/acetone 5:5), 0.16 (hexane/AcOEt 7:3). $[\alpha]_{\mathbf{D}}+18\left(c 0.25, \mathrm{CHCl}_{3}, 27{ }^{\circ} \mathrm{C}\right)$; IR (film) 3421 (br m), 2931 (m), 2857 (m), 1589 (w), 1471 (m), 1427 (m), 1390 (w), 1361 (w), 1225 (w), 1110 (s), 1006 (m) cm ${ }^{-1}$; ESMS (ES ) m/z (\%) $407.3\left((\mathrm{M}+\mathrm{Na})^{+}, 100\right) ;{ }^{1} \mathbf{H}$ NMR $\left(400 \mathrm{MHz}, \mathrm{CDCl}_{3}\right) \delta$ 7.71-7.62 ( $\left.4 \mathrm{H}, \mathrm{m}\right)$, 7.49-7.39 (6 H, m), $5.71(1 \mathrm{H}, \mathrm{d}, J=5.0 \mathrm{~Hz}), 4.83(1 \mathrm{H}, \mathrm{d}, J=3.5 \mathrm{~Hz}), 4.48(1 \mathrm{H}, \mathrm{dt}, J=7.0,9.0 \mathrm{~Hz}), 4.12(1$ H, dd, $J=4.0,10.0 \mathrm{~Hz}), 3.97(1 \mathrm{H}, \mathrm{d}, J=10.0 \mathrm{~Hz}), 3.69(1 \mathrm{H}, \mathrm{dd}, J=7.0,9.0 \mathrm{~Hz}), 3.48(1 \mathrm{H}$, $\mathrm{t}, J=8.5 \mathrm{~Hz}), 2.61(1 \mathrm{H}, \mathrm{dd}, J=5.0,9.0 \mathrm{~Hz}), 1.92(1 \mathrm{H}, \mathrm{s}), 1.11(9 \mathrm{H}, \mathrm{s}) \mathrm{ppm} ;{ }^{13} \mathbf{C} \mathbf{~ N M R}+$ DEPT $\left(100 \mathrm{MHz}, \mathrm{CDCl}_{3}\right) \delta 136.0(4 \mathrm{CH}), 133.4(2 \mathrm{C}), 130.6(2 \mathrm{CH}), 128.4,128.3(4 \mathrm{CH})$, 109.1 (CH), $77.7\left(\mathrm{CH}_{2}\right), 72.98\left(\mathrm{CH}_{2}\right), 72.89(\mathrm{CH}), 71.6(\mathrm{CH}), 56.1(\mathrm{CH}), 27.3\left(3 \mathrm{CH}_{3}\right), 19.5$ (C) ppm; ESMS m/z (\%) $402.3\left(\left(\mathrm{MNH}_{4}\right)^{+}, 60\right), 407.3\left((\mathrm{MNa})^{+}, 100\right), 791.5\left((2 \mathrm{MNa})^{+}, 7\right)$; HRMS (CI) for $\mathrm{C}_{22} \mathrm{H}_{28} \mathrm{NaO}_{4} \mathrm{Si}(\mathrm{M}+\mathrm{Na})^{+}$calcd 407.1655, found 407.1652. 


\section{X-ray of 5}

Crystal data for 5: $\mathrm{C}_{6} \mathrm{H}_{10} \mathrm{O}_{4}, \mathrm{Mr}=146.14, \mathrm{~T}=120(2) \mathrm{K}$, monoclinic, space group $P 2_{1}, a=$ $6.415(1), b=5.5133(9), c=9.3553(14) \AA, \beta=99.436(8)^{\circ}, V=326.40(9) \AA^{3}, \rho_{\text {calc }}=1.487 \mathrm{~g}$ $\mathrm{cm}^{-3}, \mu=0.126 \mathrm{~mm}^{-1}, \mathrm{Z}=2$, reflections collected: 3056 , independent reflections: $807\left(R_{\text {int }}=\right.$ 0.056 ), final $R$ indices $[I>2 \sigma I]: R 1=0.0422, w R 2=0.0952, R$ indices (all data): $R 1=$ $0.0569 . w R 2=0.1024$.

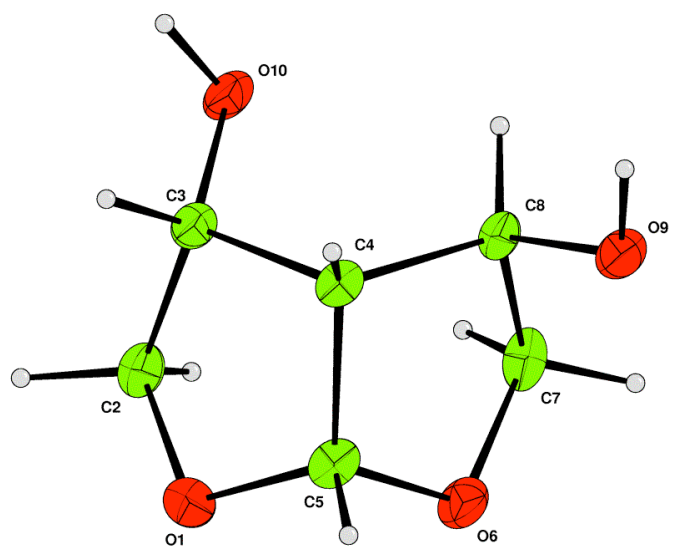




\section{Stereochemical confirmation of $\mathbf{1 7}$}

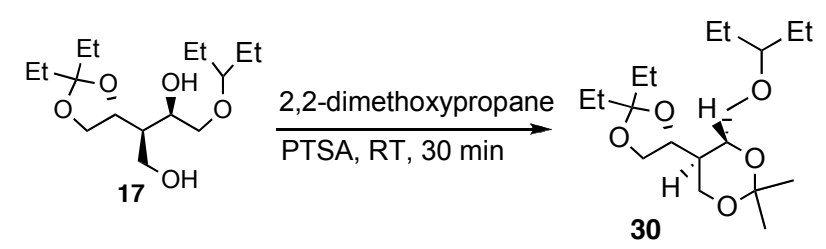

To the diol 17 (179 $\mathrm{mg}, 0.59 \mathrm{mmol})$ in 2,2-dimethoxypropane (10 mL) was added paratoluene sulfonic acid $(11 \mathrm{mg}, 0.06 \mathrm{mmol})$. The reaction mixture was stirred under a nitrogen atmosphere at room temperature for $30 \mathrm{~min}$ then $0.5 \mathrm{~mL}$ of triethylamine was added and the solvent was coevaporated with toluene to give a colorless residue. Purification by column chromatography (hexane/ EtOAc 95:5) gave the title compound as a colourless oil (171 mg, 88\%).

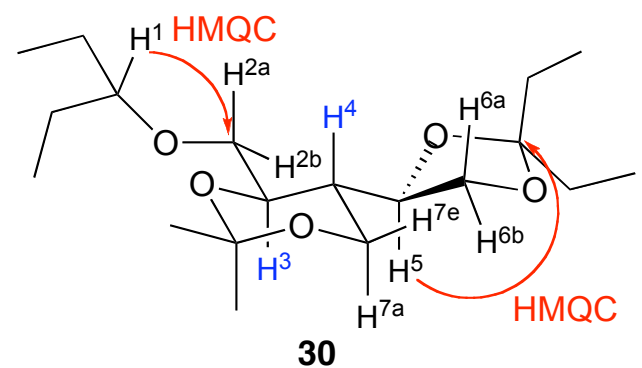

HMQC analysis allowed assignment of $\mathrm{C}^{2}$ and $\mathrm{H}^{5}$, which, after HMBC analysis, lead to the assignment of $\mathrm{H}^{2 \mathrm{a}, 2 \mathrm{~b}}$. Further analysis of the ${ }^{1} \mathrm{H}$ NMR spectrum revealed ${J_{\mathrm{H}}}^{3}{ }_{-\mathrm{H}}^{4}$ to be $9.5 \mathrm{~Hz}$, which amounts to an axial-axial coupling (see (a) Marshall, J. A.; Wang, X.-J., J. Org. Chem. 1992, 57, 1242. (b) Barrett, A. G. M.; Rys, D. J. J. Chem. Soc. Perkin trans I 1995, 1009. (c) Ghosh, A. K.; Kim, J.-H. Org. Lett. 2003, 5, 1063.)

\begin{tabular}{|c|c|c|c|c|}
\hline Proton & Shift (ppm) & Solvent & Signal & Irradiation experiments \\
\hline $\mathrm{H}^{2 \mathrm{a}}$ & 3.52 & Acetone $\mathrm{d}_{6}$ & $\begin{array}{c}\mathrm{dd}: J_{\mathrm{H}}{ }^{2 \mathrm{a}}{ }^{2}{ }^{2 \mathrm{~b}}=10.7 \mathrm{~Hz} \\
J_{\mathrm{H}}{ }^{2 \mathrm{a}}-{ }^{3}=5.7 \mathrm{~Hz}\end{array}$ & $\begin{array}{l}\text { Simplifies to } \mathrm{d}(J=10.7 \mathrm{~Hz}) \text { by } \\
\text { irradiation at } 3.86 \mathrm{ppm}\left(\mathrm{H}^{3}\right)\end{array}$ \\
\hline $\mathrm{H}^{3}$ & 3.85 & Acetone $\mathrm{d}_{6}$ & $\begin{array}{r}\text { ddd : } J_{\mathrm{H}}^{3}{ }^{3}{ }^{2 \mathrm{~b}}=3.0 \mathrm{~Hz} \\
J_{\mathrm{H}}{ }^{3}{ }^{2 \mathrm{a}}=5.5 \mathrm{~Hz} \\
J_{\mathrm{H}}{ }^{3}-{ }^{4}{ }^{4}=9.5 \mathrm{~Hz}\end{array}$ & $\begin{array}{l}\text { Simplifies to dt }(J=2.0,9.4 \mathrm{~Hz}) \\
\text { by irradiation at } 3.52 \mathrm{ppm}\left(\mathrm{H}^{2 \mathrm{a}}\right)\end{array}$ \\
\hline $\mathrm{H}^{4}$ & 2.04 & $\mathrm{CDCl}_{3}$ & $\begin{aligned} & \operatorname{tdd}: J_{\mathrm{H}}^{4}-\mathrm{H}^{3}=9.5 \mathrm{~Hz} \\
& J_{\mathrm{H}}{ }^{4}{ }^{5}=7.0 \mathrm{~Hz} \\
& J_{\mathrm{H}}{ }^{4}-\mathrm{H}=5.5 \mathrm{~Hz} \\
& J_{\mathrm{H}}{ }^{4}-\mathrm{H}=7.3 \mathrm{~Hz}\end{aligned}$ & $\begin{array}{l}\text { Simplifies to ddd }(J=9.4,7.1 \text {, } \\
5.5 \mathrm{~Hz}) \text { by irradiation at } 4.17 \\
\text { ppm }\left(\mathrm{H}^{5}\right)\end{array}$ \\
\hline & 2.02 & Acetone $\mathrm{d}_{6}$ & & $\begin{array}{l}\text { Simplifies to td }(J=7.3,4.7 \mathrm{~Hz}) \\
\text { by irradiation at } 3.86 \mathrm{ppm}\left(\mathrm{H}^{3}\right)\end{array}$ \\
\hline $\mathrm{H}^{5}$ & 4.17 & $\mathrm{CDCl}_{3}$ & $\begin{array}{r}\text { ddd }: J_{\mathrm{H}-\mathrm{H}^{5}}^{5}=6.0 \mathrm{~Hz} \\
J_{\mathrm{H}}^{5-\mathrm{b}}=8.6 \mathrm{~Hz} \\
J_{\mathrm{H}-\mathrm{H}}^{5}=7.0 \mathrm{~Hz}\end{array}$ & $\begin{array}{l}\text { Simplifies to dd }(J=8.6,6.0 \mathrm{~Hz}) \\
\text { by irradiation at } 2.04 \mathrm{ppm}\left(\mathrm{H}^{4}\right)\end{array}$ \\
\hline
\end{tabular}




\section{X-ray structures of the paranitrophenolates of $25 \mathrm{~b}, 26 \mathrm{~b}$}

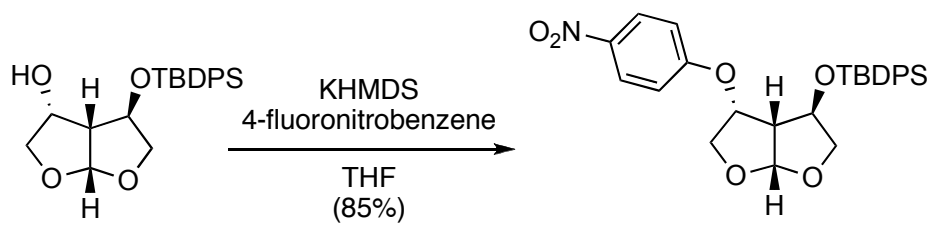

$25 b$<smiles>O=[N+]([O-])c1ccc(O[C@@H]2CO[C@@H]3OC[C@H](OS(=O)(=O)[O-])[C@@H]23)cc1</smiles>

$26 b$

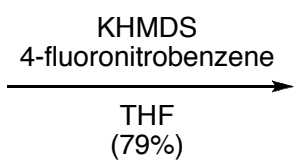

32

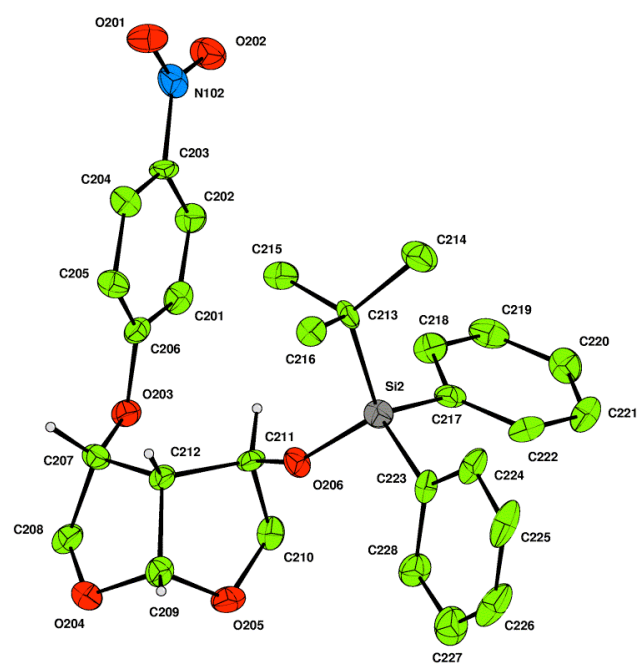

31

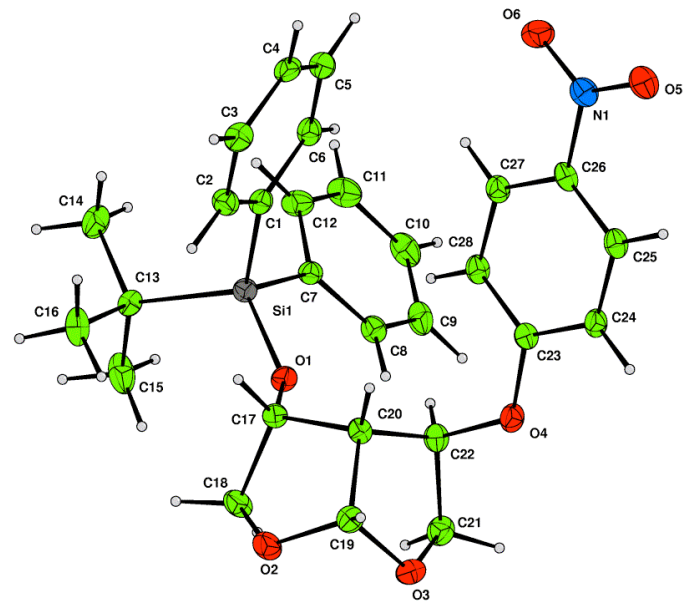

32

Thermal ellipsoids drawn at the 35\% probability level, Thermal ellipsoids drawn at the $35 \%$ probability level. only stereo centre hydrogen atoms shown for clarity.

Crystal data for $31 \mathrm{C}_{28} \mathrm{H}_{31} \mathrm{NO}_{6} \mathrm{Si}, \mathrm{Mr}=505.63, \mathrm{~T}=$ $120(2) \mathrm{K}$, orthorhombic, space group $P 22_{1} 2_{1} 2_{1}, a=$ 8.9140(6), $b=14.6373(17), c=39.801(5) \AA, V=$ $5193.2(9) \AA^{3}, \rho_{\text {calc }}=1.293 \mathrm{~g} \mathrm{~cm}^{-3}, \mu=0.133 \mathrm{~mm}^{-1}, \mathrm{Z}$ $=8$, reflections collected: 18146, independent reflections: $4807\left(R_{\text {int }}=0.1484\right)$, final $R$ indices $[I>$ $2 \sigma l$ ]: $R 1=0.0697, w R 2=0.1228, R$ indices (all data): $R 1=0.2051 . w R 2=0.1635$.
Crystal data for $32 \mathrm{C}_{28} \mathrm{H}_{31} \mathrm{NO}_{6} \mathrm{Si}, \mathrm{Mr}=505.63, \mathrm{~T}=$ $120(2) \mathrm{K}$, monoclinic, space group $P 2_{1}, a=9.0127(2)$, $b=9.2099(2), c=15.7159(3) \AA, \beta=104.151(1)^{\circ}, V=$ $1264.93(5) \AA^{3}, \rho_{\text {calc }}=1.328 \mathrm{~g} \mathrm{~cm}^{-3}, \mu=0.137 \mathrm{~mm}^{-1}, \mathrm{Z}$ $=2$, reflections collected: 18341, independent reflections: $5689\left(R_{\mathrm{int}}=0.0663\right)$, final $R$ indices $[I>$ $2 \sigma l: R 1=0.0493, w R 2=0.1021, R$ indices (all data): $R 1=0.1040 . w R 2=0.1259$. 
V. Copies of ${ }^{1} \mathrm{H}$ and ${ }^{13} \mathrm{C}$ NMR spectra of 5, 16, 17, 25a, 26b
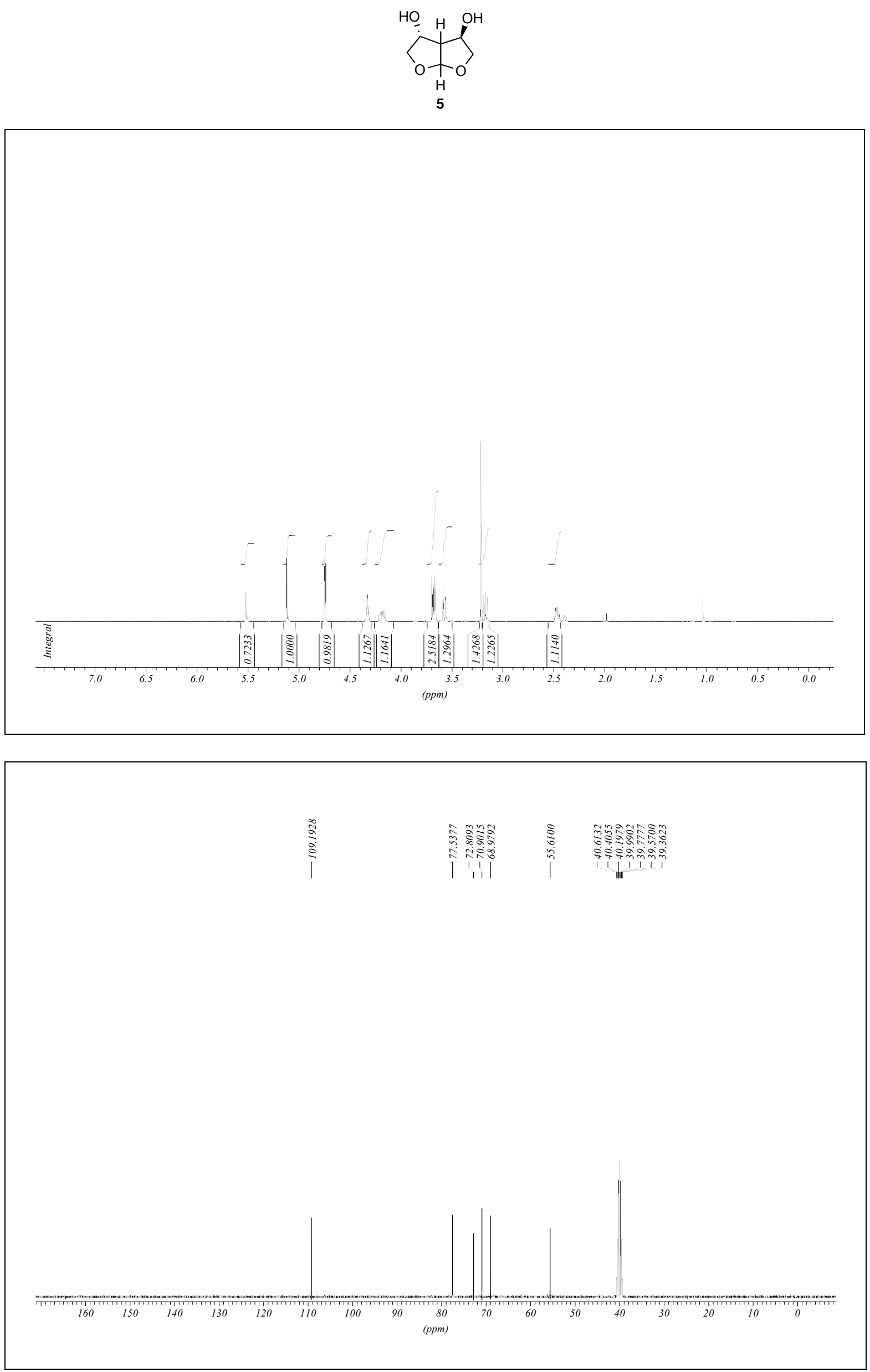

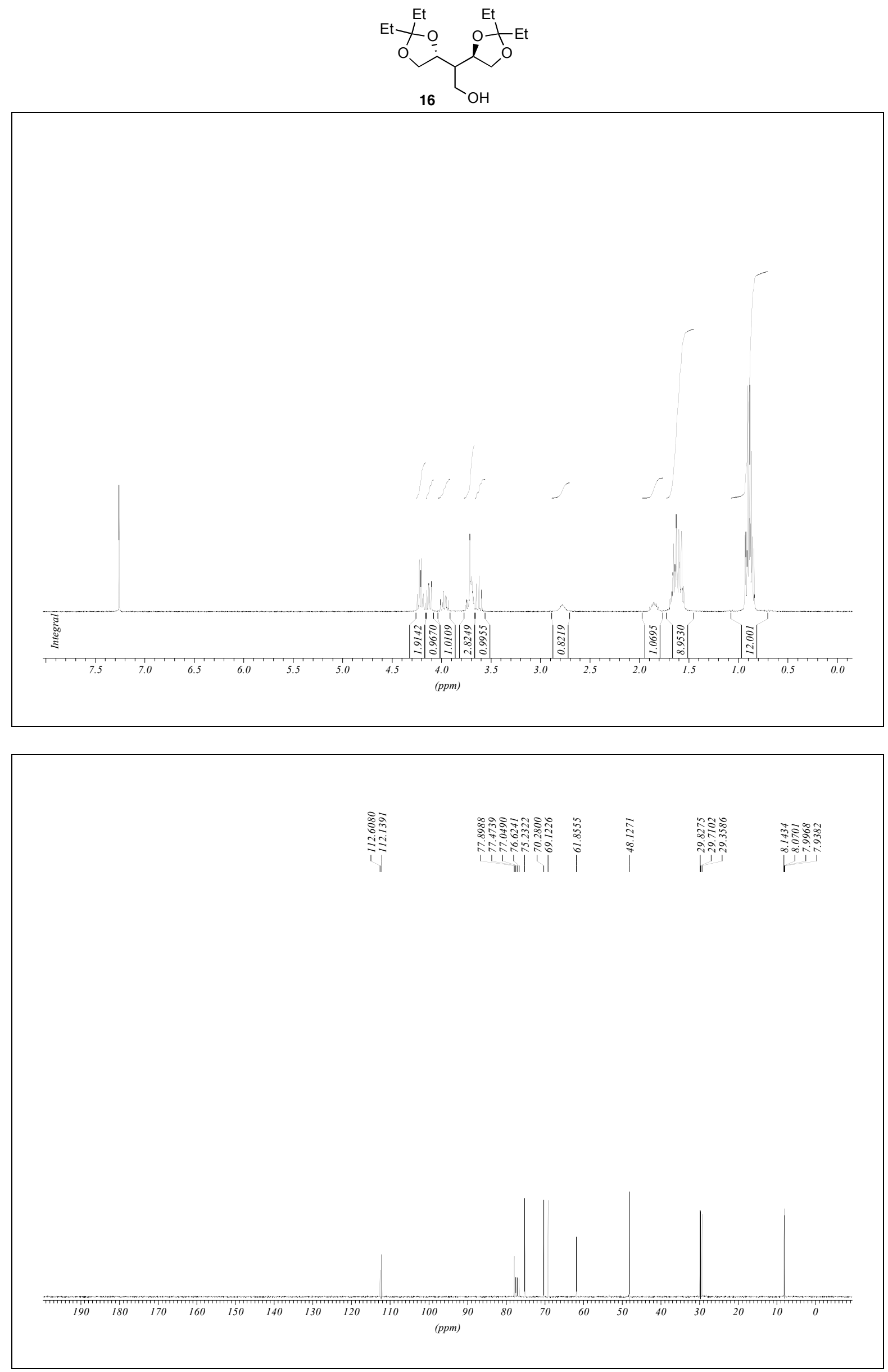

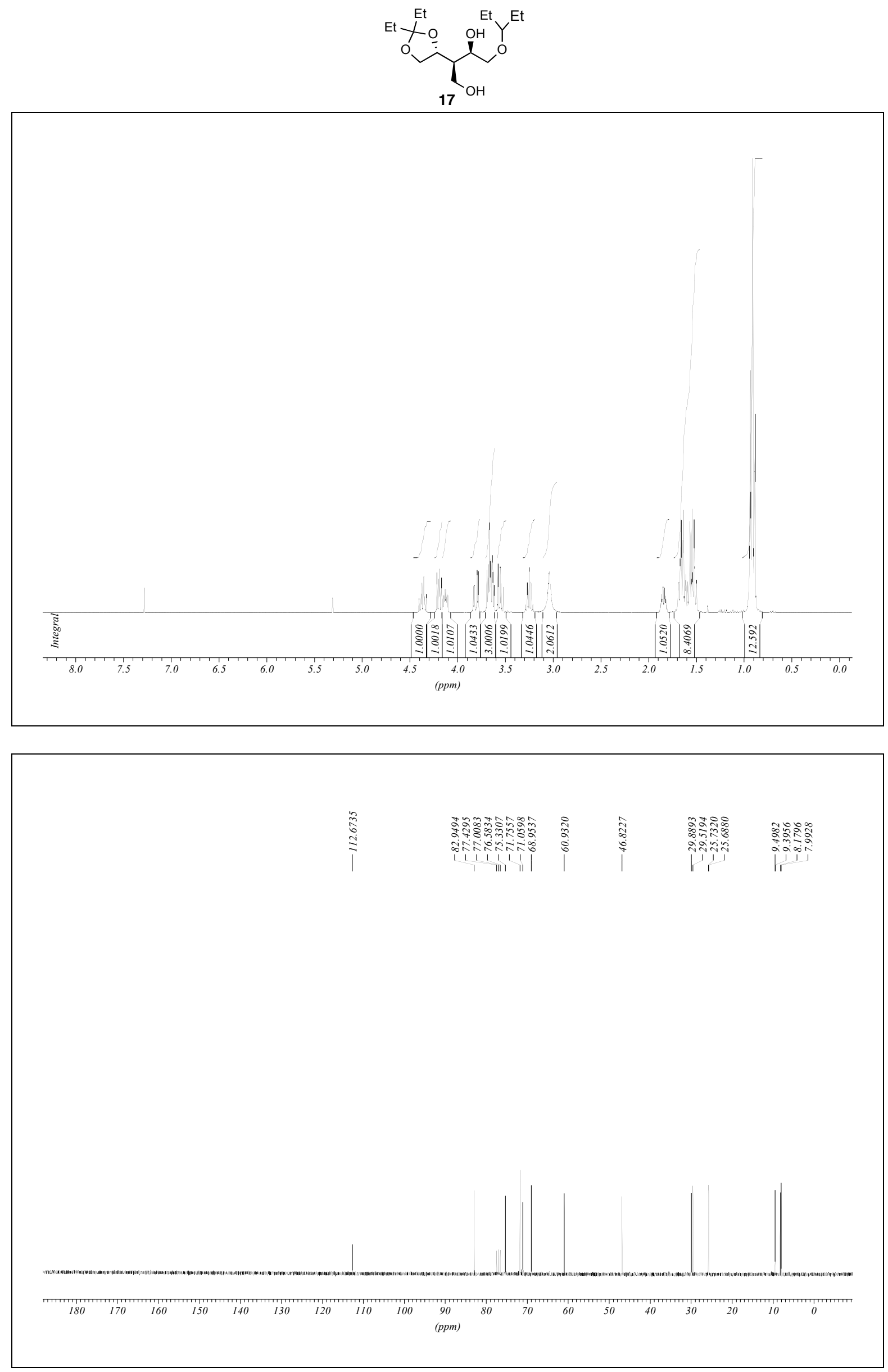

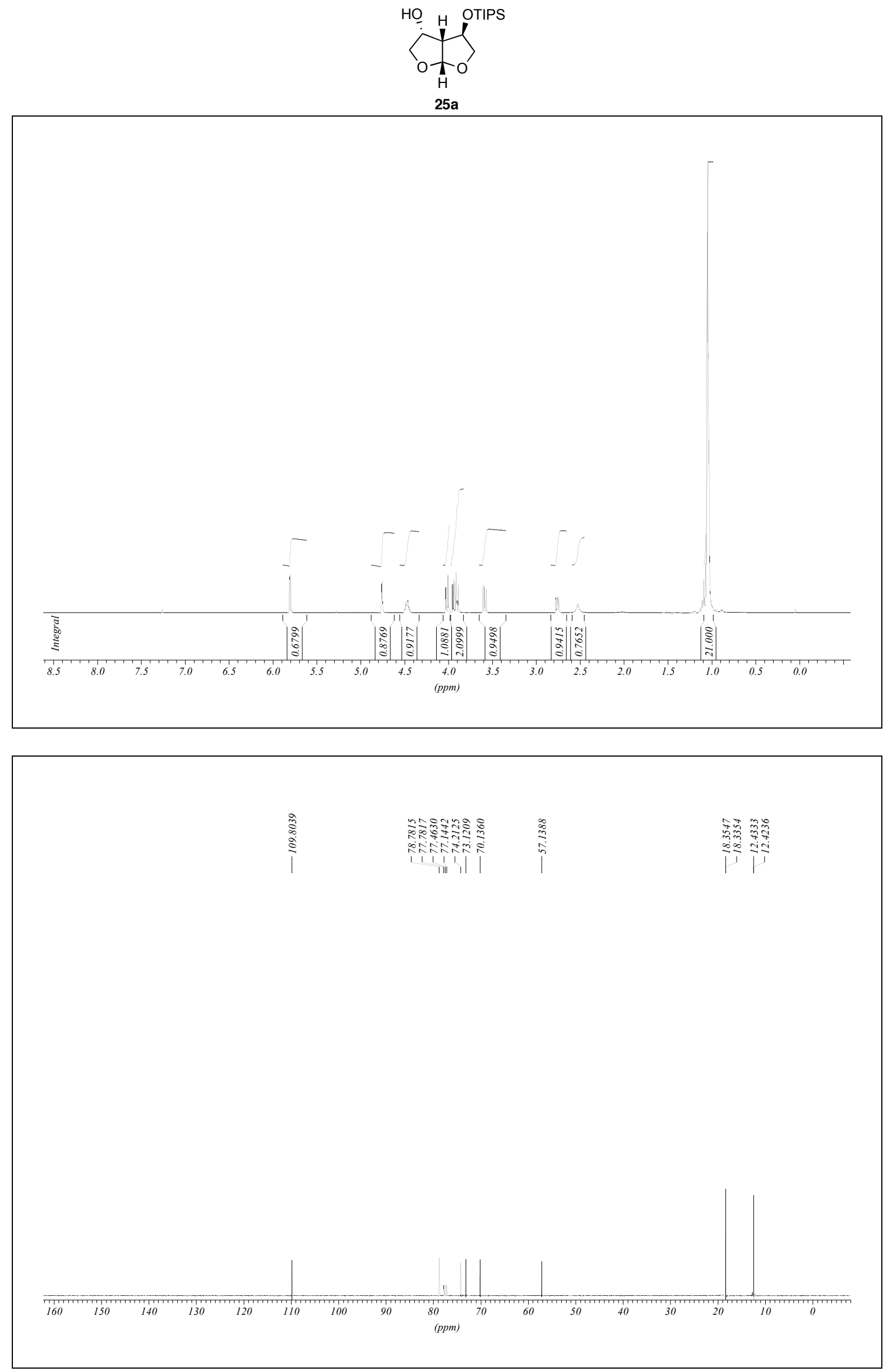


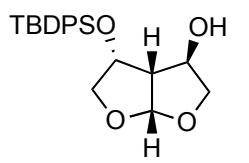

26b
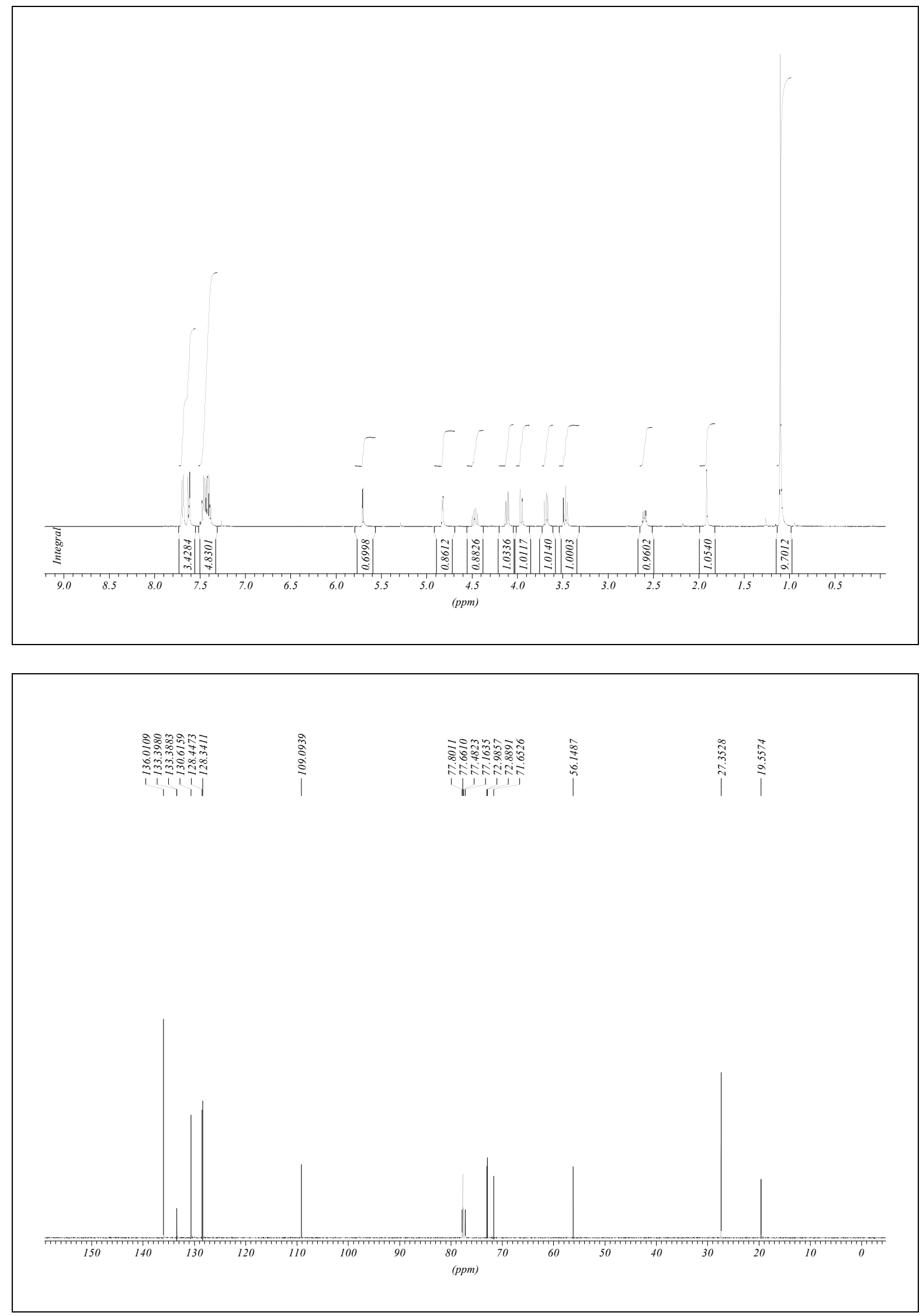